\title{
Microplastics as both a Sink and a Source of Bisphenol A in the Marine Environment
}

Xuemin Liu, ${ }^{\dagger}$ Huahong Shi, ${ }^{\ddagger}$ Bing Xie, ${ }^{\dagger}$ Dionysios D. Dionysiou, ${ }^{\S}{ }^{*}$ and Yaping Zhao ${ }^{\dagger, *}$

$4{ }^{\dagger}$ School of Ecological and Environmental Sciences, Shanghai Key Laboratory for Urban Ecological

5 Process and Eco-Restoration, East China Normal University, Shanghai 200241, China

$6 \quad$ State Key Laboratory of Estuarine and Coastal Research, East China Normal University, Shanghai, $7 \quad 200241$, China

8 Environmental Engineering and Science Program, Department of Chemical and Environmental

9 Engineering (DChEE), 705 Engineering Research Center, University of Cincinnati, Cincinnati, Ohio, USA

11 The supporting information included the following items:

12 BPA detection method

13 Calculation of Hysteresis index (HI)

14 Temporal Patterns of BPA Sorption on MPs.

15 Number of Pages: 14

16 Number of Figures: 5

17 Number of Tables: 7 
BPA Detection Method. The BPA concentration was analyzed by a Waters Acquity ${ }^{\mathrm{TM}}$

ultrahigh performance liquid chromatography-tandem mass spectrometer (UHPLC-MS/MS) equipped with an HSS T3 $(2.1 \mathrm{~mm} \times 100 \mathrm{~mm}, 1.7 \mu \mathrm{m})$ column using the selective ion monitoring (SIM) mode. The column temperature was set at $40{ }^{\circ} \mathrm{C}$ and the injection volume was $4 \mu \mathrm{L}$. The mobile phase A, B was ultrapure water and acetonitrile-methanol $(1: 2, \mathrm{v} / \mathrm{v})$ with flow rate of $0.4 \mathrm{ml} \mathrm{min} \mathrm{m}^{-1}$, respectively. The mobile phase gradient was programed as $45 \% \mathrm{~A}(0 \mathrm{~min}), 50 \% \mathrm{~A}(7.6 \mathrm{~min}), 100 \% \mathrm{~A}(8 \mathrm{~min})$ and finally $45 \% \mathrm{~A}(10 \mathrm{~min})$. A mass spectrometric analysis was conducted on a Waters triple quadrupole tandem mass spectrometer with a Z-spray electrospray interface (Waters Corp., Manchester, UK). BPA was measured in negative ion mode $[\mathrm{M}-\mathrm{H}]^{-}$with mother/daughter ions of $227 / 145$. The flow rate and temperature of the desolvation gas $\left(\mathrm{N}_{2}\right)$ were $800 \mathrm{~L} \mathrm{~h}^{-1}$ and $500{ }^{\circ} \mathrm{C}$, respectively. The flow rate of the collision gas (Ar) was $0.17 \mathrm{ml} \mathrm{min}^{-1}$, and the capillary voltage was $2.8 \mathrm{~V}$. The recovery of BPA was $96.3 \%$ with standard deviation (SD) below $5.5 \%$. Limit of detection was $0.3 \mathrm{ng} \mathrm{L}^{-1}$. The analysis of reagent blanks $(\mathrm{n}=3)$ revealed that the whole experimental and analytical system were free of BPA contamination. All experiments were conducted in triplicate.

Calculation of Hysteresis Index (HI). The hysteresis index (HI) was calculated using the following equation:

$$
\mathrm{HI}=\frac{\mathrm{Q}_{\mathrm{e}}^{\mathrm{d}}-\mathrm{Q}_{\mathrm{e}}^{\mathrm{s}}}{\mathrm{Q}_{\mathrm{e}}^{\mathrm{S}}} \mid \mathrm{T}, \mathrm{C}_{\mathrm{e}}
$$

Where $\mathrm{T}$ and $\mathrm{C}_{\mathrm{e}}$ are experimental temperature and equilibrium concentrations of solute, respectively. $\mathrm{Q}_{\mathrm{e}}^{\mathrm{d}}$ represents the equilibrium solute concentration on MPs in desorption system, and $\mathrm{Q}_{\mathrm{e}}^{\mathrm{s}}$ is the calculated solute concentration from $\mathrm{C}_{\mathrm{e}}$ assuming that desorption 
process is completely reversible. The HI values were positively correlated with the degree of desorption hysteresis. HI value is 0 when desorption is completely reversible.

Temporal Patterns of BPA Sorption on MPs. Equilibrium sorption kinetics experiments of BPA on MPs were conducted to give insight into the interaction between MPs and BPA at equilibrium state (Figure S1). Owing to the hydrogen bonding-dominating sorption mechanism, PA and PU were rapidly associated with BPA and reach equilibrium within 24 hours. In the first 10 minutes, the sorption capacity of BPA on PA and PU can reach $97.4 \%$ and $99.1 \%$ of the equilibrium sorption capacity, respectively. For the other 10 kinds of MPs, the equilibrium state was reached in 5 days. Glassy plastics such as PS, PVC, PMMA, and PET exhibit a relatively rapid sorption towards BPA than the rubbery PEs and PP. Diffusion of BPA molecules into the plastic matrix is expected based upon the flexible structure and greater free volumes of PEs and PP. The chains of glassy PS, PC, PVC, PMMA and PET are "frozen" at room temperature which does not facilitate diffusion into the material as expect for PE and PP. Therefore, faster saturation was observed on glassy plastics compared with rubbery plastics. Rochman's work suggested that glassy PET and PVC reach equilibrium much faster than rubbery LDPE, HDPE, and PP for PAHs and PCBs. ${ }^{1}$ Moreover, uncertainties in diffusion length caused by film thickness or particle diameters could also affect the equilibrium time. ${ }^{2,3}$ Smaller particle size may also play a role in promoting faster sorption of HDPE, PS, and PA compared with other plastics. In the real environments, the sorption equilibrium state of HOCs on MPs will be more unpredictable and complex. According to a field investigation conducted by Rochman et al., it takes at least 6 months or longer time for PCBs and PAHs to reach sorption equilibrium within HDPE, LDPE and PP, which was much longer than that obtained in laboratory study. ${ }^{1}$ This can probably ascribe to plastics' weathering that would increase the diffusivity and effective sorption sites. ${ }^{4,5}$ 


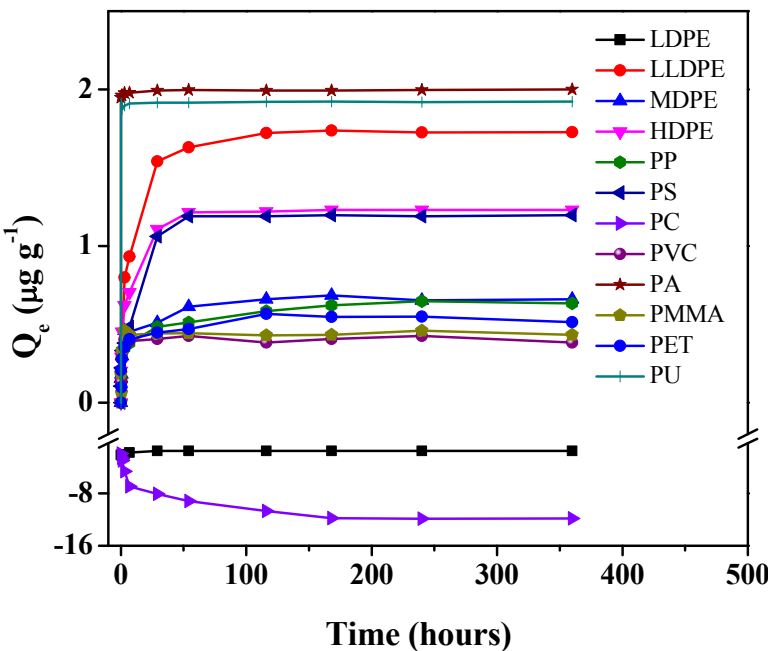

Figure S1. The sorption kinetics of BPA on MPs.

81

82

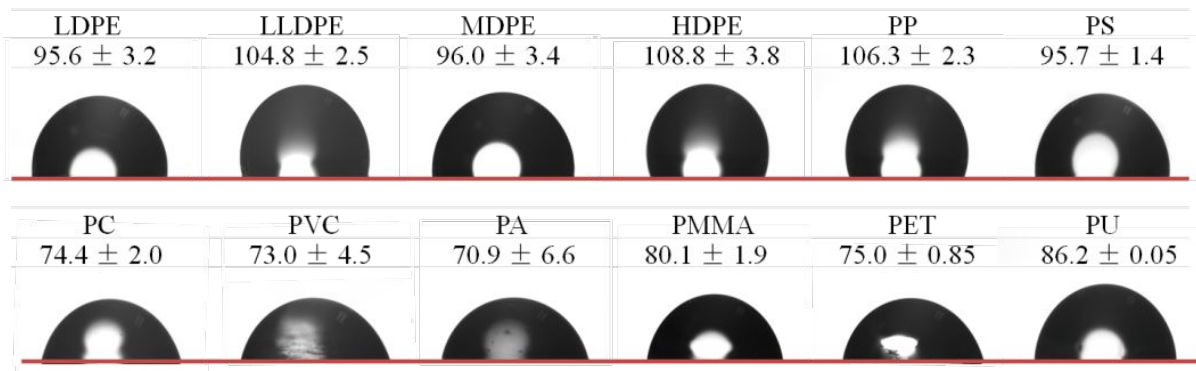

Figure S2. Contact angle images for selected MPs.

85 

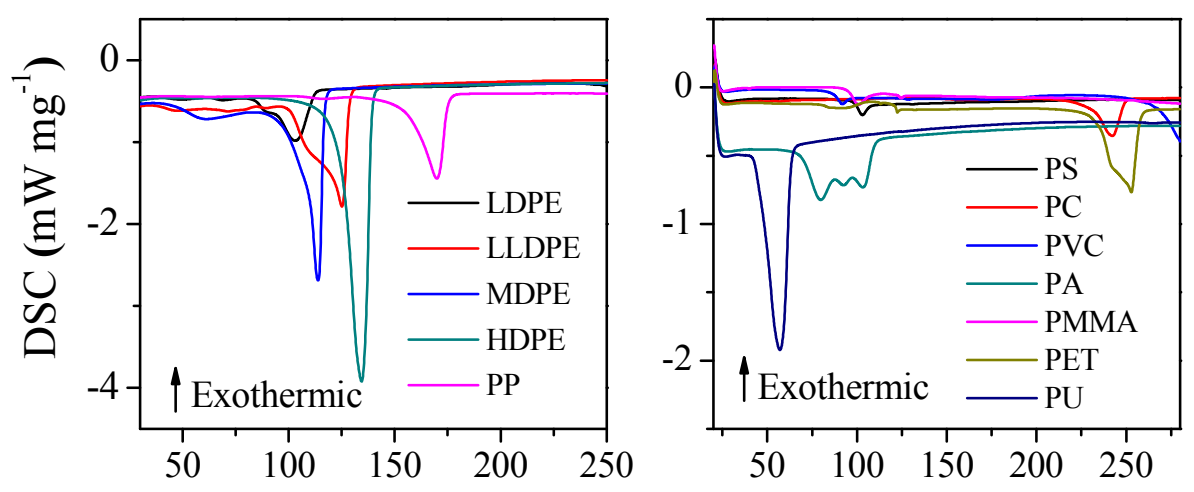

91

Temperature $\left({ }^{\circ} \mathrm{C}\right)$

92 Figure S3. Differential scanning calorimeter spectra of selected MPs

93

94 Table S1. Properties and compositions of the different water matrices (mg L-1).

\begin{tabular}{|c|c|c|c|c|}
\hline & Ultrapure water & Yangtze River water & Simulated water & Seawater \\
\hline $\mathrm{pH}$ & 7.1 & 8.0 & 7.8 & 7.9 \\
\hline Salinity (\%) & 0 & 0 & 3.4 & 3.5 \\
\hline TOC & 0 & 2.4 & 0 & 3.6 \\
\hline $\mathrm{Na}^{+}$ & - & 9.0 & $1.0 \times 10^{4}$ & $8.9 \times 10^{3}$ \\
\hline $\mathrm{K}^{+}$ & - & 9.0 & - & $3.4 \times 10^{2}$ \\
\hline $\mathrm{Ca}^{2+}$ & - & $3.3 \times 10$ & - & $5.0 \times 10^{2}$ \\
\hline $\mathrm{Mg}^{2+}$ & - & 5.4 & $3.6 \times 10^{2}$ & $1.2 \times 10^{3}$ \\
\hline $\mathrm{Cl}^{-}$ & - & $1.0 \times 10$ & $1.6 \times 10^{4}$ & $1.8 \times 10^{4}$ \\
\hline $\mathrm{NO}_{3}^{-}$ & - & 1.41 & - & 1.5 \\
\hline $\mathrm{SO}_{4}{ }^{2-}$ & - & $3.3 \times 10$ & $1.2 \times 10^{3}$ & $2.3 \times 10^{3}$ \\
\hline $\mathrm{PO}_{4}^{3-}$ & - & - & - & - \\
\hline
\end{tabular}


Table S2. The properties of selected MPs.

\begin{tabular}{|c|c|c|c|c|c|c|c|c|c|c|c|c|}
\hline Type & $\begin{array}{l}\text { Molecular } \\
\text { formula }\end{array}$ & $\begin{array}{l}\text { Density } \\
\left(\mathrm{g} \mathrm{cm}^{-3}\right)\end{array}$ & $\begin{array}{l}\text { Particle } \\
\text { size } \\
\text { range } \\
(\mu \mathrm{m})\end{array}$ & $\begin{array}{l}\text { Mean particle } \\
\text { Size }(\mu \mathrm{m})\end{array}$ & $\begin{array}{l}\mathbf{S}_{\text {area }} \\
\left(\mathbf{m}^{2} \mathbf{g}^{-1}\right)\end{array}$ & $\begin{array}{l}V_{\text {Pore }} \\
\left(\mathbf{1 0}^{-3} \mathrm{~cm}^{3} \mathrm{~g}^{-1}\right)\end{array}$ & $\begin{array}{l}T_{m} \\
\left({ }^{\circ} \mathrm{C}\right)\end{array}$ & $\begin{array}{l}T_{g} \\
\left({ }^{\circ} \mathrm{C}\right)\end{array}$ & $\begin{array}{l}\text { Contact } \\
\text { Angle } \\
\left({ }^{\circ}\right)\end{array}$ & $\begin{array}{l}\text { Crystallinity } \\
\text { (\%) }\end{array}$ & Structural formula & $\begin{array}{l}\text { Microscopic } \\
\text { images }\end{array}$ \\
\hline PET & $\left(\mathrm{C}_{10} \mathrm{H}_{6} \mathrm{O}_{4}\right)_{\mathrm{n}}$ & 1.38 & $<15 \mu \mathrm{m}$ & 10.0 & 0.487 & 0.420 & 250 & 80.0 & $75.0 \pm 0.85$ & 43.2 & & \\
\hline PU & $\left(\mathrm{C}_{17} \mathrm{H}_{16} \mathrm{~N}_{2} \mathrm{O}_{4}\right)_{\mathrm{n}}$ & 1.25 & $<50 \mu \mathrm{m}$ & 25.4 & 0.951 & 0.520 & 70 & 120.0 & $86.2 \pm 0.05$ & 20.0 & & \\
\hline
\end{tabular}

$\mathrm{S}_{\text {area: }}$ Surface area; $\mathrm{T}_{\mathrm{m}}$ : Melting Point;

$\mathrm{T}_{\mathrm{g}}$ : Glass transition temperatureat which a plastic's amorphous fraction changes from glassy to rubbery. It was obtained through differential scanning calorimetry (Figure S3). The PEs and PP can be viewed as rubbery polymers at room temperature due to their $\mathrm{T}_{\mathrm{g}} \mathrm{s}$ being below room temperature. The others can be viewed as glassy polymers.

Crystallinity: It describes the abundance of amorphous and crystalline domains in a plastic. It was estimated using differential scanning calorimetry (Figure S3, Table S1). 
Table S3. (a) Correlations analysis.

\begin{tabular}{|c|c|c|c|c|c|c|}
\hline & & $\mathbf{Q}_{\mathbf{e}}$ & Crystallinity & $\begin{array}{l}\text { Water contact } \\
\text { angle } \\
\text { (hydrophobicity) }\end{array}$ & $\begin{array}{l}\text { Particle } \\
\text { size }\end{array}$ & $\begin{array}{l}\text { Surface } \\
\text { area }\end{array}$ \\
\hline $\begin{array}{l}\text { Pearson } \\
\text { correlation } \\
\text { coefficient }\end{array}$ & $\begin{array}{l}\mathrm{Q}_{\mathrm{e}} \\
\text { Crystallinity } \\
\text { Water contact angle } \\
\text { ( hydrophobicity) } \\
\text { Particle size } \\
\text { Surface area }\end{array}$ & $\begin{array}{l}1.000 \\
0.312 \\
0.647 \\
0.052 \\
-0.472\end{array}$ & $\begin{array}{l}0.312 \\
1.000 \\
0.599 \\
-0.004 \\
-0.161\end{array}$ & $\begin{array}{l}0.647 \\
0.599 \\
1.000 \\
0.209 \\
-0.100\end{array}$ & $\begin{array}{l}0.052 \\
-0.004 \\
0.209 \\
1.000 \\
-0.290\end{array}$ & $\begin{array}{l}-0.100 \\
-0.100 \\
1.000\end{array}$ \\
\hline $\begin{array}{l}p \quad \text { value } \\
\text { (1-tailed) }\end{array}$ & $\begin{array}{l}\mathrm{Q}_{\mathrm{e}} \\
\text { Crystallinity } \\
\text { Hydrophobicity } \\
\text { Particle size } \\
\text { Surface area }\end{array}$ & $\begin{array}{l}\cdot \\
0.226 \\
0.042^{*} \\
0.451 \\
0.119\end{array}$ & $\begin{array}{l}0.226 \\
\cdot \\
0.050^{*} \\
0.496 \\
0.351\end{array}$ & $\begin{array}{l}0.042^{*} \\
0.050^{*} \\
\cdot \\
0.310 \\
0.407\end{array}$ & $\begin{array}{l}0.451 \\
0.496 \\
0.310 \\
\cdot \\
0.243\end{array}$ & $\begin{array}{l}0.119 \\
0.351 \\
0.407 \\
0.243\end{array}$ \\
\hline
\end{tabular}

99 (b) Coefficients of regression models predicting $\mathrm{Q}_{\mathrm{e}}$.

\begin{tabular}{|c|c|c|c|c|c|c|c|}
\hline \multirow{2}{*}{ Model } & \multicolumn{2}{|c|}{$\begin{array}{l}\text { Unstandardized } \\
\text { Coefficients }\end{array}$} & \multirow[t]{2}{*}{$\begin{array}{l}\text { Standardized } \\
\text { Coefficients } \\
\end{array}$} & \multirow{2}{*}{ t } & \multirow{2}{*}{ Sig. } & \multicolumn{2}{|c|}{ Interval for B } \\
\hline & B & $\begin{array}{l}\text { Std. } \\
\text { Error }\end{array}$ & & & & $\begin{array}{l}\text { Lower } \\
\text { Bound }\end{array}$ & $\begin{array}{l}\text { Upper } \\
\text { Bound }\end{array}$ \\
\hline (Constant) & -6.18 & 6.08 & & -1.02 & 0.384 & -25.5 & 13.2 \\
\hline crystallinity & -0.025 & 0.043 & -0.251 & -0.584 & 0.600 & -0.164 & 0.113 \\
\hline $\begin{array}{l}\text { water contact angle } \\
\text { (hydrophobicity) }\end{array}$ & 0.136 & 0.073 & 0.801 & 1.85 & 0.161 & -0.098 & 0.369 \\
\hline Particle size & -0.004 & 0.005 & -0.264 & -0.727 & 0.520 & -0.020 & 0.013 \\
\hline Surface area & -3.89 & 2.71 & -0.509 & -1.44 & 0.247 & -12.5 & 4.74 \\
\hline
\end{tabular}


Table S4. T-tests for the significance of differences in hydrogen-bond interaction and $\mathrm{Q}_{\mathrm{e}}$ for BPA sorption with selected MPs.

a. Group Statistics.

\begin{tabular}{llccclc}
\hline hydrogen-bond & N & Mean & Std. Deviation & Std. & Error \\
& & & & & Mean & \\
\hline \multirow{2}{*}{$\mathrm{Q}_{\mathrm{e}} \quad$ without } & 8 & 2.96 & 2.43 & 0.858 \\
& with & 2 & 19.6 & 0.584 & 0.413 & \\
\hline
\end{tabular}

104

105

b. Independent Samples Test.

\begin{tabular}{|c|c|c|c|c|c|c|c|c|c|c|}
\hline & \multicolumn{2}{|c|}{$\begin{array}{l}\text { Levene's } \\
\text { Test for } \\
\text { Equality of } \\
\text { Variances }\end{array}$} & \multicolumn{7}{|c|}{ t-test for Equality of Means } \\
\hline & & \multirow[t]{2}{*}{$\mathrm{F}$} & \multirow[t]{2}{*}{ Sig. } & \multirow[t]{2}{*}{$\mathrm{t}$} & \multirow[t]{2}{*}{ df } & \multirow[t]{2}{*}{$\begin{array}{l}\text { Sig. } \\
\text { (2-tailed) }\end{array}$} & \multirow[t]{2}{*}{$\begin{array}{l}\text { Mean } \\
\text { Difference }\end{array}$} & \multirow[t]{2}{*}{$\begin{array}{l}\text { Std. Error } \\
\text { Difference }\end{array}$} & \multicolumn{2}{|c|}{$\begin{array}{l}95 \% \text { Confidence } \\
\text { Interval of the } \\
\text { Difference }\end{array}$} \\
\hline & & & & & & & & & Lower & Upper \\
\hline $\mathrm{Q}_{\mathrm{e}}$ & $\begin{array}{l}\text { Equal variances } \\
\text { assumed } \\
\text { Equal variances } \\
\text { not assumed }\end{array}$ & 6.15 & 0.038 & $\begin{array}{l}-9.22 \\
-17.4\end{array}$ & $\begin{array}{l}8 \\
7.72\end{array}$ & $\begin{array}{l}0.00^{* *} \\
0.00^{* *}\end{array}$ & $\begin{array}{l}-16.6 \\
-16.6\end{array}$ & 1.80 & -20.8 & -12.5 \\
\hline
\end{tabular}


Table S5. T-tests of the significance of the differences in aggregation state and $\mathrm{Q}_{\mathrm{e}}$ for BPA

114 sorption on selected MPs.

a. Group Statistics.

\begin{tabular}{lllllll}
\hline \multirow{2}{*}{ aggregation } & N & Mean & Std. Deviation & Std. & Error \\
& & & & Mean & \\
\hline \multirow{2}{*}{$\mathrm{Q}_{\mathrm{e}}$} & glassy plastics & 3 & 0.805 & 0.715 & 0.413 \\
& rubbery plastics & 4 & 3.72 & 2.01 & 1.01 & \\
\hline
\end{tabular}

116

b. Independent Samples Test.

\begin{tabular}{|c|c|c|c|c|c|c|c|c|c|c|}
\hline & \multicolumn{2}{|c|}{$\begin{array}{l}\text { Levene's } \\
\text { Test for } \\
\text { Equality of } \\
\text { Variances }\end{array}$} & \multicolumn{7}{|c|}{ t-test for Equality of Means } \\
\hline & & \multirow[t]{2}{*}{$\mathrm{F}$} & \multirow[t]{2}{*}{ Sig. } & \multirow[t]{2}{*}{$\mathrm{t}$} & \multirow[t]{2}{*}{ df } & \multirow[t]{2}{*}{$\begin{array}{l}\text { Sig. } \\
\text { (2-tailed) }\end{array}$} & \multirow[t]{2}{*}{$\begin{array}{l}\text { Mean } \\
\text { Difference }\end{array}$} & \multirow[t]{2}{*}{$\begin{array}{l}\text { Std. Error } \\
\text { Difference }\end{array}$} & \multicolumn{2}{|c|}{$\begin{array}{l}95 \% \text { Confidence } \\
\text { Interval of the } \\
\text { Difference }\end{array}$} \\
\hline & & & & & & & & & Lower & Upper \\
\hline $\mathrm{Q}_{\mathrm{e}}$ & $\begin{array}{l}\text { Equal variances } \\
\text { assumed } \\
\text { Equal variances } \\
\text { not assumed }\end{array}$ & 3.01 & 0.144 & $\begin{array}{l}-2.36 \\
-2.69\end{array}$ & $\begin{array}{l}5 \\
3.93\end{array}$ & $\begin{array}{l}0.045^{*} \\
0.036^{*}\end{array}$ & $\begin{array}{l}-2.92 \\
-2.92\end{array}$ & 1.24 & $\begin{array}{l}-6.10 \\
-5.95\end{array}$ & $\begin{array}{l}0.262 \\
0.116\end{array}$ \\
\hline
\end{tabular}


Table S6. Equations predicting $\mathrm{Q}_{\mathrm{T}}, \mathrm{Q}_{\mathrm{P}}$ and $\mathrm{Q}_{\mathrm{A}}$ for the sorption of BPA on MPs.

\begin{tabular}{|c|c|c|c|c|c|}
\hline MPs & $Q_{\mathrm{T}}\left(\mu \mathrm{g} \mathrm{g}^{-1}\right)$ & $\mathbf{R}^{2}$ & $Q_{p}\left(\mu g^{-1}\right)$ & $Q_{A}\left(\mu g^{-1}\right)$ & $K_{d}\left(L_{k g}^{-1}\right)$ \\
\hline LLDPE & $\mathrm{Q}_{\mathrm{T}}=0.697 \mathrm{C}_{\mathrm{e}}^{0.345}$ & 0.957 & $\mathrm{Q}_{\mathrm{P}}=0.0065 \mathrm{C}_{\mathrm{e}}$ & $\mathrm{Q}_{\mathrm{A}}=0.697 \mathrm{C}_{\mathrm{e}}^{0.345-0.0065 \mathrm{C}_{\mathrm{e}}}$ & 13.8 \\
\hline MDPE & $\mathrm{Q}_{\mathrm{T}}=0.046 \mathrm{C}_{\mathrm{e}}^{0.711}$ & 0.998 & $\mathrm{Q}_{\mathrm{P}}=0.0050 \mathrm{C}_{\mathrm{e}}$ & $\mathrm{Q}_{\mathrm{A}}=0.046 \mathrm{C}_{\mathrm{e}}^{0.711}-0.0050 \mathrm{C}_{\mathrm{e}}$ & 8.0 \\
\hline HDPE & $\mathrm{Q}_{\mathrm{T}}=0.273 \mathrm{C}_{\mathrm{e}}^{0.508}$ & 0.990 & $\mathrm{Q}_{\mathrm{P}}=0.0100 \mathrm{C}_{\mathrm{e}}$ & $\mathrm{Q}_{\mathrm{A}}=0.273 \mathrm{C}_{\mathrm{e}}^{0.508}-0.0100 \mathrm{C}_{\mathrm{e}}$ & 14.8 \\
\hline PP & $\mathrm{Q}_{\mathrm{T}}=0.079 \mathrm{C}_{\mathrm{e}}^{0.427}$ & 0.934 & $\mathrm{Q}_{\mathrm{P}}=0.0015 \mathrm{C}_{\mathrm{e}}$ & $\mathrm{Q}_{\mathrm{A}}=0.079 \mathrm{C}_{\mathrm{e}}^{0.427}-0.0015 \mathrm{C}_{\mathrm{e}}$ & 2.2 \\
\hline PS & $\mathrm{Q}_{\mathrm{T}}=0.695 \mathrm{C}_{\mathrm{e}}^{0.385}$ & 0.949 & $\mathrm{Q}_{\mathrm{P}}=0.0103 \mathrm{C}_{\mathrm{e}}$ & $\mathrm{Q}_{\mathrm{A}}=0.695 \mathrm{C}_{\mathrm{e}}^{0.385}-0.0103 \mathrm{C}_{\mathrm{e}}$ & 18.8 \\
\hline PVC & $\mathrm{Q}_{\mathrm{T}}=0.036 \mathrm{C}_{\mathrm{e}}^{0.425}$ & 0.886 & $\mathrm{Q}_{\mathrm{P}}=0.00042 \mathrm{C}_{\mathrm{e}}$ & $\mathrm{Q}_{\mathrm{A}}=0.036 \mathrm{C}_{\mathrm{e}}^{0.425}-0.00042 \mathrm{C}_{\mathrm{e}}$ & 0.4 \\
\hline PA & $\mathrm{Q}_{\mathrm{T}}=67.0 \mathrm{C}_{\mathrm{e}}^{0.903}$ & 0.982 & $\mathrm{Q}_{\mathrm{P}}=73.1 \mathrm{C}_{\mathrm{e}}$ & $\mathrm{Q}_{\mathrm{A}}=67.0 \mathrm{C}_{\mathrm{e}}^{0.903-73.1 \mathrm{C}_{\mathrm{e}}}$ & 76,287 \\
\hline PMMA & $\mathrm{Q}_{\mathrm{T}}=0.086 \mathrm{C}_{\mathrm{e}}^{0.333}$ & 0.927 & $\mathrm{Q}_{\mathrm{P}}=0.00044 \mathrm{C}_{\mathrm{e}}$ & $\mathrm{Q}_{\mathrm{A}}=0.086 \mathrm{C}_{\mathrm{e}}^{0.333}-0.00044 \mathrm{C}_{\mathrm{e}}$ & 1.3 \\
\hline PET & $\mathrm{Q}_{\mathrm{T}}=0.017 \mathrm{C}_{\mathrm{e}}^{0.741}$ & 0.986 & $\mathrm{Q}_{\mathrm{P}}=0.0029 \mathrm{C}_{\mathrm{e}}$ & $\mathrm{Q}_{\mathrm{A}}=0.017 \mathrm{C}_{\mathrm{e}}^{0.741}-0.0029 \mathrm{C}_{\mathrm{e}}$ & 3.5 \\
\hline PU & $\mathrm{Q}_{\mathrm{T}}=1.06 \mathrm{C}_{\mathrm{e}}{ }^{0.953}$ & 0.992 & $\mathrm{Q}_{\mathrm{P}}=0.870 \mathrm{C}_{\mathrm{e}}$ & $\mathrm{Q}_{\mathrm{A}}=1.06 \mathrm{C}_{\mathrm{e}}^{0.953}-0.870 \mathrm{C}_{\mathrm{e}}$ & 916 \\
\hline
\end{tabular}

125

126

127
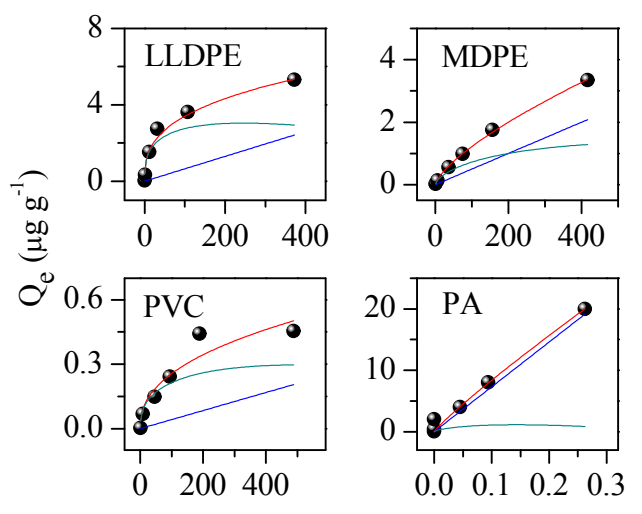

$\begin{array}{lllll}0.0 & 0.1 & 0.2 & 0.3\end{array}$
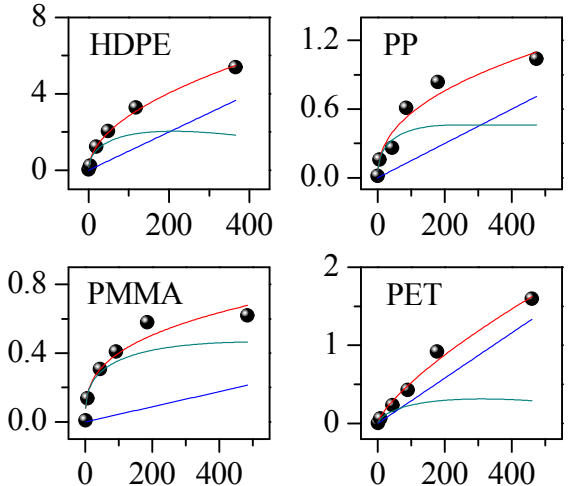

$\mathrm{C}_{\mathrm{e}}(\mu \mathrm{g} / \mathrm{L})$
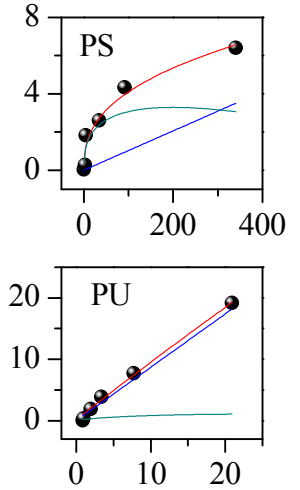

Figure S4. The relevant models predicting $\mathrm{Q}_{\mathrm{T}}$ (red lines), $\mathrm{Q}_{\mathrm{P}}$ (blue lines) and $\mathrm{Q}_{\mathrm{A}}$ (cyan lines) for BPA sorption by various MPs. 

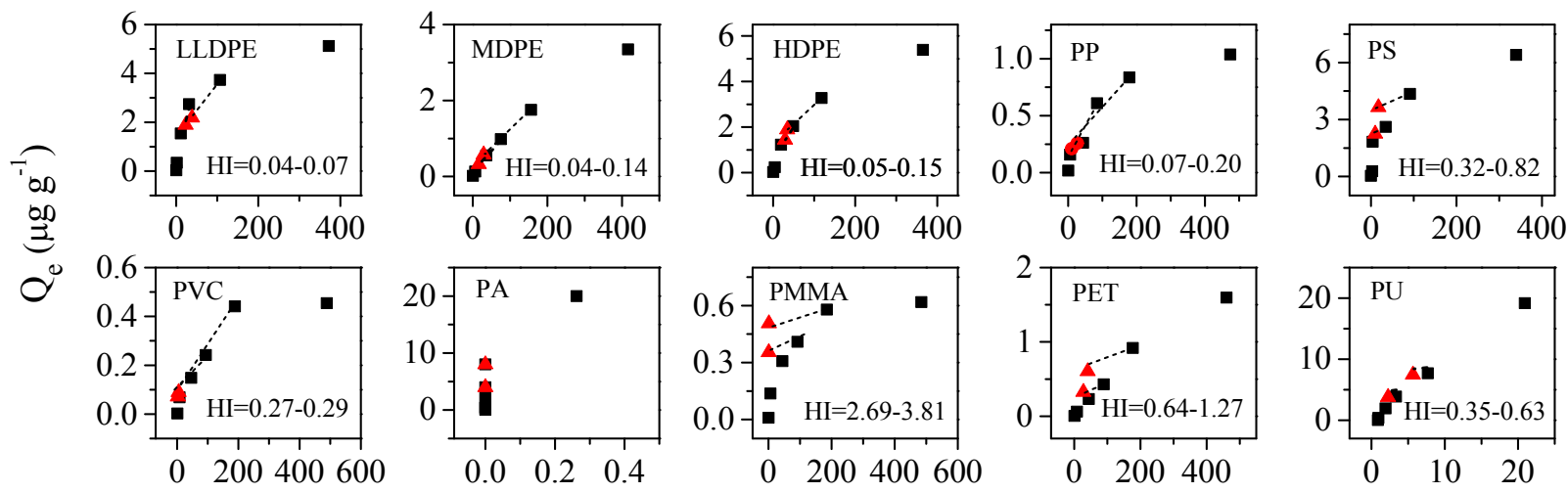

Figure S5. Sorption-desorption isotherms of BPA with different polymers. Two desorption data points were obtained for each polymer and hysteresis indices (HIs) were calculated using Liu's equation (please refer to the supplementary information). ${ }^{18}$ 
Table S7. BPA desorbed from various MPs compared with aqueous or dietary exposure standards.

\begin{tabular}{|c|c|c|c|c|c|c|c|c|c|}
\hline \multirow{2}{*}{$\begin{array}{l}\text { Medium/ } \\
\text { uptake }\end{array}$} & \multirow{2}{*}{ Polymer } & \multirow{2}{*}{$\begin{array}{l}C_{B P A} \\
\left(\mu g L^{-1}\right)\end{array}$} & \multirow{2}{*}{$\begin{array}{l}C_{\text {BPA }}^{\prime} \\
\left(n g L^{-1}\right)\end{array}$} & \multicolumn{2}{|l|}{ Zebra Danio } & \multicolumn{2}{|c|}{ Japanese Medaka } & \multirow{2}{*}{$\begin{array}{l}\text { Sea Bass } \\
\begin{array}{l}\mathrm{EC}_{10}=9120 \mathrm{ng} \mathrm{L}^{-1} \\
\text { (vitellogenin, } \\
\text { day period }^{9} \text { ) }\end{array}\end{array}$} & Xiphophorushelleri \\
\hline & & & & $\begin{array}{l}\mathrm{EC}_{10}=6.5 \mathrm{mg} \mathrm{L}^{-1} \\
(\mathrm{embryo} \text { toxicity, } \\
\left.2 \text { day period }^{6}\right)\end{array}$ & $\begin{array}{l}\mathrm{LOEC}=15 \mu \mathrm{g} \mathrm{L}^{-1} \\
(\mathrm{DNA} \text { methylation, } \\
7 \text { day period }^{7} \text { ) }\end{array}$ & $\begin{array}{l}\mathrm{EC}_{10}=5.2 \mathrm{mg} \mathrm{L}^{-1} \\
\left(\mathrm{embryo}^{-} \text {toxicity, }\right. \\
\left.7 \text { day period }^{6}\right)\end{array}$ & $\begin{array}{l}\text { LOEC }=100 \mathrm{ng} \mathrm{L}^{-1} \\
\text { (immune gene } \\
\begin{array}{l}\text { expression, } 60 \text { day } \\
\text { period }^{8} \text { ) }\end{array}\end{array}$ & & $\begin{array}{l}\text { LOEC }=200 \text { ng L }^{-1} \\
\left(\text { growth, }^{\prime} \quad 4 \text { day }\right. \\
\left.\text { period }^{10}\right)\end{array}$ \\
\hline \multirow{10}{*}{$\begin{array}{l}\text { seawater } \\
\text { /aqueous }\end{array}$} & LLDPE & 3.97 & 2.48 & $<\mathrm{EC}_{10}$ & $<\mathrm{LOEC}$ & $<\mathrm{EC}_{10}$ & $<\mathrm{LOEC}$ & $<\mathrm{EC}_{10}$ & $<$ LOEC \\
\hline & MDPE & 2.11 & 1.32 & $<\mathrm{EC}_{10}$ & $<$ LOEC & $<\mathrm{EC}_{10}$ & $<$ LOEC & $<\mathrm{EC}_{10}$ & $<$ LOEC \\
\hline & HDPE & 8.17 & 5.10 & $<\mathrm{EC}_{10}$ & $<$ LOEC & $<\mathrm{EC}_{10}$ & $<$ LOEC & $<\mathrm{EC}_{10}$ & $<$ LOEC \\
\hline & PP & 4.86 & 3.03 & $<\mathrm{EC}_{10}$ & $<$ LOEC & $<\mathrm{EC}_{10}$ & $<$ LOEC & $<\mathrm{EC}_{10}$ & $<$ LOEC \\
\hline & PS & 2.23 & 1.39 & $<\mathrm{EC}_{10}$ & $<$ LOEC & $<\mathrm{EC}_{10}$ & $<$ LOEC & $<\mathrm{EC}_{10}$ & $<$ LOEC \\
\hline & PVC & 4.60 & 2.87 & $<\mathrm{EC}_{10}$ & $<$ LOEC & $<\mathrm{EC}_{10}$ & $<$ LOEC & $<\mathrm{EC}_{10}$ & $<$ LOEC \\
\hline & $\mathrm{PA}$ & 0 & 0 & $<\mathrm{EC}_{10}$ & $<\mathrm{LOEC}$ & $<\mathrm{EC}_{10}$ & $<\mathrm{LOEC}$ & $<\mathrm{EC}_{10}$ & $<$ LOEC \\
\hline & PMMA & 3.42 & 2.13 & $<\mathrm{EC}_{10}$ & $<\mathrm{LOEC}$ & $<\mathrm{EC}_{10}$ & $<\mathrm{LOEC}$ & $<\mathrm{EC}_{10}$ & $<\mathrm{LOEC}$ \\
\hline & PET & 8.03 & 5.01 & $<\mathrm{EC}_{10}$ & $<$ LOEC & $<\mathrm{EC}_{10}$ & $<$ LOEC & $<\mathrm{EC}_{10}$ & $<$ LOEC \\
\hline & PU & 0 & 0 & $<\mathrm{EC}_{10}$ & $<$ LOEC & $<\mathrm{EC}_{10}$ & $<$ LOEC & $<\mathrm{EC}_{10}$ & $<$ LOEC \\
\hline seawater & LDPE & 57.3 & 35.7 & $<\mathrm{EC}_{10}$ & $<$ LOEC & $<\mathrm{EC}_{10}$ & $<$ LOEC & $<\mathrm{EC}_{10}$ & $<$ LOEC \\
\hline /aqueous & $\mathrm{PC}$ & 276.6 & $172.6 \mathrm{c}$ & $<\mathrm{EC}_{10}$ & $<$ LOEC & $<\mathrm{EC}_{10}$ & $>$ LOEC & $<\mathrm{EC}_{10}$ & $<$ LOEC \\
\hline $15 \mathrm{mM} \mathrm{ST}$ & LDPE & 8.49 & 5.30 & $<\mathrm{EC}_{10}$ & $<\mathrm{LOEC}$ & $<\mathrm{EC}_{10}$ & $<$ LOEC & $<\mathrm{EC}_{10}$ & $<\mathrm{LOEC}$ \\
\hline /ingestion & $\mathrm{PC}$ & 17.4 & 10.9 & $<\mathrm{EC}_{10}$ & $<$ LOEC & $<\mathrm{EC}_{10}$ & $<$ LOEC & $<\mathrm{EC}_{10}$ & $<$ LOEC \\
\hline
\end{tabular}

Note: $1 . \mathrm{C}_{\mathrm{BPA}}$ is the release observed from $0.5 \mathrm{~g}$ of the MPs in $20 \mathrm{~mL}$ of the solution (i.e., $25 \mathrm{~g}$ of the particles in $1 \mathrm{~L}$ of the solution).

2. $\mathrm{C}_{\mathrm{BPA}}^{\prime}$ is the predicted released of BPA in an actual aqueous environment. ${ }^{11,12}$

3. EC: effect concentration; LOEC: lowest observed effect concentration. 


\section{References}

1. Rochman, C. M.; Hoh, E.; Hentschel, B. T.; Kaye, S., Long-term field measurement of sorption of organic contaminants to five types of plastic pellets: implications for plastic marine debris. Environ Sci Technol 2013, $47,1646-1654$

2. Sangam, H. P.; Rowe, R. K., Migration of dilute aqueous organic pollutants through HDPE geomembranes. Geotext Geomembranes 2001, 19, 329-357.

3. Teuten, E. L.; Saquing, J. M.; Knappe, D. R.; Barlaz, M. A.; Jonsson, S.; Bjorn, A.; Rowland, S. J.; Thompson, R. C.; Galloway, T. S.; Yamashita, R.; Ochi, D.; Watanuki, Y.; Moore, C.; Viet, P. H.; Tana, T. S.; Prudente, M.; Boonyatumanond, R.; Zakaria, M. P.; Akkhavong, K.; Ogata, Y.; Hirai, H.; Iwasa, S.; Mizukawa, K.; Hagino, Y.; Imamura, A.; Saha, M.; Takada, H., Transport and release of chemicals from plastics to the environment and to wildlife. Philos Trans R Soc Lond B Biol Sci 2009, 364, 2027-2045.

4. Mato, Y.; Isobe, T.; Takada, H.; Kanehiro, H.; Ohtake, C.; Kaminuma, T., Plastic resin pellets as a transport medium for toxic chemicals in the marine environment. Environ Sci Technol 2001, 35, 318-324.

5. Wang, J.; Tan, Z.; Peng, J.; Qiu, Q.; Li, M., The behaviors of microplastics in the marine environment. Mar Environ Res 2016,113, 7-17.

6. Schiller, V.; Zhang, X.; Hecker, M.; Schafers, C.; Fischer, R.; Fenske, M., Species-specific considerations in using the fish embryo test as an alternative to identify endocrine disruption. Aquat Toxicol 2014, 155, 62-72.

7. Liu, Y.; Zhang, Y.; Tao, S.; Guan, Y.; Zhang, T.; Wang, Z., Global DNA methylation in gonads of adult zebrafish Danio rerio under bisphenol A exposure. Ecotoxicol Environ Saf 2016, 130, 124-132.

8. Qiu, W.; Shen, Y.; Pan, C.; Liu, S.; Wu, M.; Yang, M.; Wang, K. J., The potential immune modulatory effect of chronic bisphenol A exposure on gene regulation in male medaka (Oryzias latipes) liver. Ecotoxicol Environ Saf 2016, 130, 146-154.

9. Correia, A. D.; Sandro, F.; Martin, S.; Goncalves, J. F.; Petra, B.; Lamoree, M. H.; Evaristo, M. A.; Reis-Henriques, M. A., Mixtures of estrogenic chemicals enhance vitellogenic response in sea bass. Environmental health perspectives 2007, 115, 115-121.

10. Kwak, H. I.; Bae, M. O.; Lee, M. H.; Lee, Y. S.; Lee, B. J.; Kang, K. S.; Chae, C. H.; Sung, H. J.; Shin, J. S.; Kim, J. H., Effects of nonylphenol, bisphenol A, and their mixture on the viviparous swordtail fish (Xiphophorus helleri). Environ Toxicol Chem 2010, 20, 787-795.

11. Zhang, K.; Shi, H. H.; Peng, J. P.; Wang, Y. H.; Xiong, X.; Wu, C. X.; Lam, P. K. S., Microplastic pollution in China's inland water systems: A review of findings, methods, characteristics, effects, and 

management. Sci Total Environ 2018, 630, 1641-1653.

193 12. Luo, W. Y.; Su, L.; Craig, N. J.; Du, F. N.; Wu, C. X.; Shi, H. H., Comparison of microplastic pollution in 194 different water bodies from urban creeks to coastal waters. Environ Pollut 2019, 246, 174-182. 